\title{
Female Polymorphism, Frequency Dependence, and Rapid Evolutionary Dynamics in Natural Populations
}

\author{
Erik I. Svensson, ${ }^{\star}$ Jessica Abbott, ${ }^{\dagger}$ and Roger Härdling ${ }^{\ddagger}$
}

Section for Animal Ecology, Ecology Building, Lund University, SE-223 62 Lund, Sweden

Submitted September 15, 2004; Accepted December 20, 2004; Electronically published March 14, 2005

ABSTRACT: Rapid evolutionary change over a few generations has been documented in natural populations. Such changes are observed as organisms invade new environments, and they are often triggered by changed interspecific interactions, such as differences in predation regimes. However, in spite of increased recognition of antagonistic male-female mating interactions, there is very limited evidence that such intraspecific interactions could cause rapid evolutionary dynamics in nature. This is because ecological and longitudinal data from natural populations have been lacking. Here we show that in a color-polymorphic damselfly species, male-female mating interactions lead to rapid evolutionary change in morph frequencies between generations. Field data and computer simulations indicate that these changes are driven by sexual conflict, in which morph fecundities are negatively affected by frequency- and density-dependent male mating harassment. These frequency-dependent processes prevent population divergence by maintaining a female polymorphism in most populations. Although these results contrast with the traditional view of how sexual conflict enhances the rate of population divergence, they are consistent with a recent theoretical model of how females may form discrete genetic clusters in response to male mating harassment.

Keywords: color polymorphism, Ischnura elegans, rapid evolution, population genetics, sexual dimorphism, sexual selection.

Rapid evolutionary dynamics on short (ecological) timescales, that is, over a few generations, have been the subject of much recent interest (Reznick et al. 1997; Huey et al. 2000; Sinervo et al. 2000). Evidence for rapid evolutionary change comes from both natural populations (Huey et al.

\footnotetext{
* Corresponding author; e-mail: erik.svensson@zooekol.lu.se.

† E-mail: jessica.abbott@zooekol.lu.se.

¥ E-mail: roger.hardling@zooekol.lu.se.
}

Am. Nat. 2005. Vol. 165, pp. 567-576. (c) 2005 by The University of Chicago. 0003-0147/2005/16505-40626\$15.00. All rights reserved.

2000; Sinervo et al. 2000) and laboratory experiments (Yoshida et al. 2003), and such short-term evolutionary dynamics are often driven by interspecific interactions such as predation (Reznick et al. 1997). In contrast, the role of intraspecific interactions has been appreciated only recently (Sinervo and Lively 1996; Sinervo et al. 2000). One powerful evolutionary force caused by intraspecific interactions is sexual conflict (Rice and Holland 1997). Theory (Parker and Partridge 1998; Gavrilets 2000; Gavrilets and Waxman 2002), data from laboratory selection experiments (Rice 1996; Martin and Hosken 2003), and comparative studies (Arnqvist 1998; Arnqvist and Rowe $2002 a, 2002 b)$ collectively suggest that conflicts between males and females over mating rates can have profound evolutionary consequences and may even be an engine of speciation (Rice and Holland 1997; Arnqvist et al. 2000).

Plant and animal species with discrete and heritable morphs have been successfully used by evolutionary biologists in the past to explore the interplay among frequency-dependent selection, genetic drift, and other evolutionary processes (Eckert and Barrett 1995; Ågren and Ericson 1996; Sinervo and Lively 1996). Genetic cycles in morph frequencies have been observed in colorpolymorphic lizards (Sinervo and Lively 1996; Sinervo et al. 2000) but have not yet been reported in other natural systems and never in the context of sexual conflict. Color morphs often differ in several morphological, physiological, and behavioral traits, and these multitrait differences are promoted by correlational selection for multiple optimal character combinations (Svensson et al. 2001a, $2001 b$; Sinervo and Svensson 2002). Many damselfly species are characterized by the presence of female color polymorphisms (Robertson 1985; Cordero 1992; Cordero et al. 1998; Andrés et al. 2000), and a genetic basis for such polymorphisms has been demonstrated in several species of the genus Ischnura (Cordero 1990). Males are monomorphic (Askew 1988) and are blue and black, whereas there are typically two or three female color morphs, including a male mimic ("androchrome" females).

Explanations for the maintenance of such polymorphisms have focused on density- and frequency-dependent 
male-female interactions and conflicts over mating rate (Robertson 1985; Cordero et al. 1998). It has been suggested that as male mimics, androchrome females suffer less from male mating harassment. Direct support for selection is equivocal; survival differences between morphs are either slight or absent (Cordero 1992; Cordero et al. 1998), and there is no evidence for intrinsic fecundity differences between morphs in the field (Fincke 1994). Population comparisons of neutral genetic variation and morph frequency variation indicate that balancing selection maintains these polymorphisms (Andrés et al. 2000, 2002; Wong et al. 2003). No study has presented data on the fluctuations in morph frequencies between generations that are expected to arise as a consequence of frequencydependent selection. Most previous field studies on Ischnura have focused on fitness differences between morphs arising from mortality (Cordero 1992; Cordero et al. 1998). However, it is more likely that selection by sexual conflict caused by excessive male mating harassment would reduce female fecundity (Holland and Rice 1999), which was one of the topics of this study.

A major limitation of field studies aiming to quantify selection is that most studies are neither spatially nor temporally replicated (Kingsolver et al. 2001; Svensson and Sinervo 2004; Svensson et al. 2004). This problem is of particular concern if selection is frequency dependent and there are no intrinsic fitness differences between the morphs. Morph-specific fitness data from one or a few populations during a single season may then fail to reveal any evidence for selection, and the average fitnesses of the different morphs may be equal on a long-term scale. To demonstrate and quantify frequency-dependent selection under such circumstances, one needs to estimate female fitness components across several different populations with different morph frequencies and relate morphspecific fitnesses to the genetic composition of the study populations. The fitness of a given morph is likely to change between years within populations in a predictable way as the population morph frequency changes (Sinervo and Lively 1996; Sinervo et al. 2000). No previous field studies in the polymorphic damselfly systems have quantified both female fitness components and morph frequencies across several years and populations and then investigated the causal links between temporal changes in morph fitnesses and temporal changes in morph frequencies.

In this article, we present results from a study on damselfly morph frequency variation between populations, morph frequency changes over several generations, and variation in female fecundity. We use data from the field to empirically estimate fecundity parameters describing the strength of frequency-dependent selection, and we then utilize these parameters in a population genetic simulation model to investigate the maintenance and dynamics of this polymorphism. The results in this study point to an important role for negative frequency-dependent selection in the dynamics of sexual conflict and suggest that this process may act as a conservative force in population divergence.

\section{Methods}

\section{Study Organism: Genetics, Ecology, and Mating System}

Ischnura elegans is a small damselfly that occurs in Europe from northern Spain to southern Sweden, and its distribution also extends further into eastern Europe and Asia (Askew 1988). Ischnura elegans overwinters in the larval stage and emerges as an adult late the following May or early June in Sweden (Askew 1988). Three female morphs have been described in this species: androchrome (A), infuscans (I), and infuscans-obsoleta (O; Cordero et al. 1998). In its sister species (Ischnura graellsii), the polymorphism is caused by an autosomal locus with three alleles in a dominance hierarchy with expression limited to females (males are monomorphic; Cordero 1990). Analyses of hybrid progeny between I. graellsii and I. elegans indicate that the three-allelic genetic basis of the color polymorphisms is identical in these two species (A. Cordero, personal communication).

Previous molecular genetic studies of I. elegans in natural populations have revealed that in the wild, most females mate with multiple males (Cooper et al. 1996). Laboratory studies on other Ischnura species have further revealed that singly mated females are not sperm limited and that females that have mated only once lay just as many fertile eggs as females that have mated several times (Sirot and Brockmann 2001). These and several other features of Ischnura mating systems suggest that the observed mating rate in the field, where females are continuously exposed to male mating attempts and male mating harassment, may be above the optimal female mating rate (Arnqvist and Nilsson 2000). Most laboratory experiments on the fitness effects of female mating rates have revealed that female longevity declines for each additional mating, whereas an optimal (intermediate) mating rate maximizes fecundity, although there is considerable variation between different taxonomic groups in these patterns (Arnqvist and Nilsson 2000). Arnqvist and Nilsson (2000) suggested that fecundity costs of mating may be higher in the field, where females are continuously exposed to male mating harassment and where food supply is more limited than in the laboratory. Mating rates in I. elegans are considerably higher than in other members of this genus (Robinson and Allgeyer 1996). Multiple matings in I. elegans are thus likely to be costly to females, and the costs in nature may 
be even higher than documented in previous laboratory studies because copulations may last for several hours, during which the female cannot obtain any food and becomes susceptible to predation (Robinson and Allgeyer 1996; Cordero et al. 1998).

In this study, we used morph-specific copulation probabilities in the field as a measure of male mating harassment, an approach that has been successfully used in other insect systems with intense premating struggles and malefemale mating conflicts (Rowe 1992). This approach was motivated by considerations of the mating system and a high degree of male mating harassment in this species, and data from previous studies showed that male preference is correlated with population morph frequencies (Van Gossum et al. 1999). Previous studies of I. elegans have revealed that the three morphs do not differ in their field survival rates, and this will create a link between morph-specific copulation frequencies and morph-specific copulation rates (Cordero et al. 1998). If males form a search image for the most common morph in the population (Van Gossum et al. 1999; Fincke 2004) and female morphs are subject to frequency-dependent male mating harassment, morph-specific copulation probabilities in the field should, at least to some degree, reflect the intensity of morphspecific male mating harassment. Male mating harassment of females may of course not always result in copulations but could instead reduce female food intake and through such a path negatively affect female fecundity. Using copulation probabilities as a measure of male mating harassment may therefore underestimate the strength of male mating harassment of females, and hence our statistical tests are likely to be conservative. Behavioral studies of $I$. elegans in laboratory settings indicate that male mating harassment of females may be frequency dependent (Van Gossum et al. 2001), but the evolutionary consequences of this have yet to be documented in natural populations.

\section{Field and Laboratory Work}

We visited a total of 22 populations in southern Sweden during the summer months (May-August) of the years 2000-2003. Most of these 22 populations were visited only once and only during the first year of the study (2000), when we started our regular sampling program. The mean number of females caught on each visit was 44.42 ( $\mathrm{SE}=4.08 ; N=59$ yearly population visits). During 2001-2003, we concentrated our sampling efforts to 13 core populations, of which 10 were visited during all four seasons and three were visited during three of the seasons. The core populations were visited during 30-60 min at regular intervals each year (mean number of visits per population and season $=3.41$; $\mathrm{SE}= \pm 0.31$ ), and the number of each morph, captured singly or in copula, was recorded. During each visit, we tried to catch as many damselflies as possible, and male densities were subsequently estimated as the number of males caught divided by catching time ( $\mathrm{min}$ ). The three different female morphs have different average emergence times in the field, with the greatest difference in emergence time being about 3 days (J. Abbott and E. Svensson, unpublished data). To avoid sampling bias of morph frequencies due to seasonal differences in emergence times of the morphs, we therefore tried to spread our sampling efforts over the entire season as much as possible. Our sampling program was designed to revisit populations with a 1-2-week interval, which is longer than the average differences in emergence times between morphs.

During 2001-2003, we estimated female fecundities in 12 of the 13 core populations. Field-caught females from populations with different morph frequencies were brought in plastic containers to an indoor laboratory. Female morph and biometric measurements (total length, abdomen length, wing length, thorax width, and width of the fourth abdominal segment) were recorded. Females were placed in ovipositoria made of plastic containers with wet filter paper on the bottom (to stimulate oviposition). Females were left in these ovipositoria for 2-3 days, after which we counted the eggs laid. Of the females that were brought into the laboratory $(N=1,000), 82.2 \%$ laid eggs. The average number of unique female egg clutches per population and year was $28.23(\mathrm{SE}=2.79)$.

Of the females that laid eggs, $89.2 \%$ were old, and $10.8 \%$ were immature, as judged by the stiffness and dryness of their wings. We investigated whether there were any age effects on fecundity by performing a general linear model (GLM) with population, year, female morph, and age as categorical factors. There were significant differences in average female fecundity between populations $(F=$ 3.491, $\mathrm{df}=14,801, P<.001)$, years $(F=4.013, \mathrm{df}=$ $2,801, P=.018)$, and morphs $(F=10.477, \mathrm{df}=2,801$, $P<.001)$ but no effects of female age $(F=2.343, \mathrm{df}=$ $1,801, P=.13)$. Thus, fecundity differences between morphs and populations reported in this article are not confounded by age differences between morphs. We use the average female fecundity and the average morph fecundity in each population and each year to estimate selection coefficients, although we weighted by population size when estimating parameters. Thus, we use annual population averages rather than individual females as data points to ensure statistical independence.

Our fecundity estimate is cross-sectional rather than longitudinal and is thus not equal to total lifetime female fecundity. Cross-sectional fitness measures are useful, however, as long as they are correlated with total fitness to some extent (Lande and Arnold 1983). Previous empirical studies on damselfly fecundities under natural con- 
Table 1: Selection coefficients for the fecundity-morph frequency relation used in the population genetic simulation model

\begin{tabular}{llcccccccc}
\hline Coefficient & $S_{\mathrm{AA}}$ & $S_{\mathrm{AI}}$ & $S_{\mathrm{AO}}$ & $S_{\mathrm{IA}}$ & $S_{\mathrm{II}}$ & $S_{\mathrm{IO}}$ & $S_{\mathrm{OA}}$ & $S_{\mathrm{OI}}$ & $S_{\mathrm{OO}}$ \\
\hline Estimate & .93 & 1.17 & .25 & 1.21 & .21 & 3.40 & 1.30 & 1.52 & -1.21 \\
SD & .008 & .020 & .042 & .017 & .044 & .091 & .042 & .109 & .224 \\
\hline
\end{tabular}

Note: All selection coefficients are significantly different from 0 (see "Results"), and the variance estimates (i.e., SD) were used to generate bootstrapped estimates of a frequency region in which the polymorphism is stable (fig. $1 A$ ). The selection coefficients are partial regression coefficients from mixture models without intercept (one model for each morph) where morph-specific relative fitnesses were regressed against annual morph frequency of each population.

ditions indicate that fecundity per clutch is indeed correlated with total lifetime fecundity (Fincke 1986; Corbet 1999). Thus, our fecundity measure, although obtained in the laboratory, will reflect the recent ecological conditions a female has experienced in the field during the days immediately preceding oviposition in the laboratory and will be influenced by recent male mating harassment, cold spells, and food intake rates. In summary, our fecundity estimate is likely to be correlated with total female fecundity and is unlikely to be confounded by laboratory conditions because females were not fed during the oviposition days. The fact that our fecundity estimates explained the maintenance of the morphs in the field (see "Results") strongly suggests that it is indeed correlated with female lifetime reproductive fitness.

\section{Statistics and Parameter Estimation}

The probability that a female was found copulating was analyzed using a generalized linear model (binomial error, logit link function), with copulating females coded as 1 and those not copulating as 0. Population morph frequency changes between years across all populations were tested with another generalized model (multinomial error, logit link function) in which the dependent variable had three different outcomes (i.e., the probability that a female belonged to one of the three morphs).

Female fecundities were analyzed using multivariate and univariate GLM, with average fecundity for each morph and population as the dependent variables and the frequencies of the three morphs as predictors. Models were weighted by sample size to take into account variation in precision in estimates from populations with unequal sample sizes. For each population and year, we calculated morph frequency, mean fecundity $(W)$, and morph-specific selection coefficients $\left(S_{\text {morph }}=W_{\text {morph }} / W\right)$. A multivariate GLM (StatSoft 2003) with the morph-specific selection coefficients $\left(S_{\mathrm{A}}, S_{\mathrm{I}}, S_{\mathrm{O}}\right)$ as dependent variables and annual population morph frequencies $\left(P_{\mathrm{A}}, P_{\mathrm{I}}, P_{\mathrm{O}}\right)$ was used to test for the occurrence of frequency-dependent selection on female fecundity. The model was a mixture model with no intercept (Draper and Smith 1998) because the inde- pendent variables are mixture ingredients $\left(P_{\mathrm{A}}+P_{\mathrm{I}}+\right.$ $P_{\mathrm{O}}=1$ and always $>0$ ). The conditions for an intercept (frequency of all three morphs $=0$ ) do not exist.

After evaluating the multivariate model, we estimated parameters (regression slopes) using univariate multiple regressions. Parameters reveal how relative morph fecundities changed in relation to population morph frequencies. Nine parameters were estimated (three for each morph), and all were significantly different from 0 (table $1)$. We use notation such as $S_{\mathrm{AI}}$ to denote the partial regression of androchrome fecundity on infuscans frequency and so on. We used these regression surfaces as fitness equations in a population genetic simulation model (table $1)$.

\section{Population Genetic Simulation Model}

We calculated fitness for each of the three alleles at the present allele frequency distribution. Allele frequencies among offspring were then predicted using three discrete selection models, one for each allele frequency (Hartl and Clark 1997). Thus, we assumed that morph frequency changes between generations were proportional to relative fitnesses at the adult stage.

The model assumes that the three color morphs are determined by one locus with three alleles $\alpha, \beta$, and $\gamma$ in a dominance hierarchy, $\alpha>\beta>\gamma$, which is consistent with the known inheritance of color morph in this system (Cordero 1990). The frequencies of $\alpha, \beta$, and $\gamma$ are denoted $p$, $q$, and $r$, respectively. Assuming Hardy-Weinberg equilibrium, the frequencies of the three color morphs can be calculated as

$$
\begin{aligned}
& P_{\mathrm{A}}=p^{2}+2 p q+2 p r \\
& P_{\mathrm{I}}=q^{2}+2 q r \\
& P_{\mathrm{O}}=r^{2}
\end{aligned}
$$

where $P_{\mathrm{A}}$ is the frequency of andochrome, $P_{\mathrm{I}}$ is the frequency of infuscans, and $P_{\mathrm{O}}$ is the frequency of infuscansobsoleta. Because the organism is annual, the frequencies 
of the morphs in the next generation are completely determined by the current relative fitnesses of each morph. The data showed that female fecundity was differentially frequency dependent for each morph, and we equate morph-specific fitness with female fecundity. Specifically, the univariate multiple regressions yield the following functions relating morph fecundities $w_{\mathrm{A}}, w_{\mathrm{I}}$, and $w_{\mathrm{O}}$ to population morph frequencies:

$$
\begin{gathered}
w_{\mathrm{A}}=\mathrm{S}_{\mathrm{AA}} P_{\mathrm{A}}+\mathrm{S}_{\mathrm{AI}} P_{\mathrm{I}}+\mathrm{S}_{\mathrm{AO}} P_{\mathrm{O}}, \\
\mathrm{w}_{\mathrm{I}}=\mathrm{S}_{\mathrm{IA}} P_{\mathrm{A}}+\mathrm{S}_{\mathrm{II}} P_{\mathrm{I}}+\mathrm{S}_{\mathrm{IO}} P_{\mathrm{O}}, \\
w_{\mathrm{O}}=\mathrm{S}_{\mathrm{OA}} P_{\mathrm{A}}+\mathrm{S}_{\mathrm{OI}} P_{\mathrm{I}}-\mathrm{S}_{\mathrm{OO}} P_{\mathrm{O}} .
\end{gathered}
$$

In the simulations described below, we limited equations (2), (3), and (4) to positive values, setting any negative fecundity value to 0 . Allele-specific fitness functions can be derived by considering the probability that a given allele is combined with each of the others and the resulting fitnesses as follows:

$$
\begin{aligned}
& W_{\alpha}=w_{\mathrm{A}}, \\
& W_{\beta}=p w_{\mathrm{A}}+(q+r) w_{\mathrm{I}}, \\
& W_{\gamma}=p w_{\mathrm{A}}+q w_{\mathrm{I}}+r w_{\mathrm{O}} .
\end{aligned}
$$

The mean fitness of the alleles is

$$
\bar{W}=p W_{\alpha}+q W_{\beta}+r W_{\gamma} .
$$

The allele frequencies in the next generation $\left(p^{\prime}, q^{\prime}, r^{\prime}\right)$ will be proportional to their relative fitness in the current generation, according to the recursion equations

$$
\begin{aligned}
& p^{\prime}=\frac{p W_{\alpha}}{\bar{W}}, \\
& q^{\prime}=\frac{q W_{\beta}}{\bar{W}}, \\
& r^{\prime}=\frac{r W_{\gamma}}{\bar{W}} .
\end{aligned}
$$

New morph frequencies are thereafter calculated from the Hardy-Weinberg relations.

\section{Incorporating Environmental Noise}

To include environmental noise in the simulations, we estimated the coefficients of variation (CV) of the residuals of the linear multiple regressions relating relative morph fecundity to population morph frequency. We incorporated environmental noise in the morph-specific selection coefficients because morph dynamics are likely to also be influenced by abiotic factors (temperature, precipitation) in addition to the deterministic force of frequency-dependent selection. Environmental noise, here defined as variance in relative morph fitness not caused by frequencydependent selection, differed significantly between the three morphs (Levene's test: $F=22.032$, df $=2,84$, $P<.001)$, being lowest for androchromes $(\mathrm{CV}=9.4 \%)$, intermediate for infuscans ( $\mathrm{CV}=22.7 \%$ ), and highest for infuscans-obsoleta $(\mathrm{CV}=38.9 \%)$. Thus, the morphspecific noise estimates then became $\mathrm{CV}_{\mathrm{A}}=0.094$, $\mathrm{CV}_{\mathrm{I}}=0.227, \mathrm{CV}_{\mathrm{O}}=0.389$. In each step of the simulation, the fecundity of each morph was calculated by adding a noise factor to the deterministic value calculated from the present morph frequencies. With noise, the fecundities of androchrome, infuscans, and infuscans-obsoleta females are thus $w_{\mathrm{A}}\left(1+X_{\mathrm{A}}\right), w_{\mathrm{I}}\left(1+X_{\mathrm{I}}\right)$, and $w_{\mathrm{O}}\left(1+X_{\mathrm{O}}\right)$, respectively, where $\quad X_{\mathrm{A}} \in N\left(0, \mathrm{CV}_{\mathrm{A}}\right), \quad X_{\mathrm{I}} \in N\left(0, \mathrm{CV}_{\mathrm{I}}\right), \quad$ and $\quad X_{\mathrm{O}} \in$ $N\left(0, \mathrm{CV}_{\mathrm{O}}\right)$.

\section{Results}

We were able to obtain data on population morph frequencies, morph-specific copulation probabilities, and morph fecundities in Ischnura elegans between 2000 and 2003 (total data $=2,621$ females). All three morphs were detected in most populations in all years, and no population was monomorphic. We used the proportion of copulating females in the field as an estimate of the degree of male mating harassment of the three different morphs (see "Methods" for motivation and details about I. elegans mating system and ecology). Across all populations and years,

Table 2: Effects of male density and frequencies of the three female morphs $(\mathrm{A}, \mathrm{I}, \mathrm{O})$ on the proportion of copulating androchrome females

\begin{tabular}{lrrrr}
\hline Variable & $\beta$ & SE & \multicolumn{1}{c}{$t$} & \multicolumn{1}{c}{$P$} \\
\hline Male density & .317 & .136 & 2.335 & .025 \\
A frequency & .463 & .127 & 3.655 & $<.001$ \\
I frequency & .397 & .094 & 4.199 & $<.001$ \\
O frequency & -.224 & .077 & -2.904 & .006 \\
\hline
\end{tabular}

Note: Multiple regression model with the proportion of copulating androchrome females as the dependent variable and male density and the morph frequency estimates as predictor variables. The model has no intercept (see "Methods" for rationale). Estimates of copulation frequencies are averages from each population and year. Standardized partial regression coefficients ( $\beta$ values) and their standard errors (SE) are shown. The model reveals that the proportion of copulating androchrome females increases with male density, androchrome frequency (i.e., own frequency), and infuscans frequency, whereas it decreases with the frequency of infuscans-obsoleta. Full model: $F=5.332$, df $=4,35$, $R^{2}=0.379, P<.001$. 
androchrome females were found less often in copula than infuscans females but not infuscans-obsoleta females $(\mathrm{A}=47 \%, \mathrm{I}=74 \%, \mathrm{O}=33 \%)$. The probability that a female was found copulating in the field was significantly affected by population $\left(\chi^{2}=261.598, \mathrm{df}=20, P<.001\right)$, year $\left(\chi^{2}=210.560, \quad \mathrm{df}=3, \quad P<.001\right)$, female color morph $\left(\chi^{2}=121.588, \mathrm{df}=2, P<.001\right)$, as well as the interaction between morph and population $\left(\chi^{2}=\right.$ $66.569, \mathrm{df}=32, P<.001)$. This indicates that male mating harassment of morphs is spatially and temporally variable.

Males may form a search image for females based not only on the visual detectability of a particular morph but also on its density or frequency in the population (Fincke 2004). In support of this, we found that the proportion of copulating androchromes (the most common morph) was significantly affected by both male density and the frequencies of the three female morphs (table 2), which is consistent with the expectation from density- and frequency-dependent male mating harassment. Density- and frequency-dependent male mating harassment rates would be expected to reduce a morph's feeding rate as that morph becomes more common and reduce its energy intake needed for egg production. Hence, sexual conflict over mating is expected to affect female fecundity (Holland and Rice 1999) in a frequency-dependent fashion that is similar to apostatic survival selection by predators on more common prey (Gendron 1987).

To investigate this, we quantified fecundities of the three different morphs and related this to the morph frequencies of the populations. A multivariate GLM revealed that the relative fecundities of the three morphs were indeed frequency dependent because all the regression slopes ( $=$ selection coefficients) were significantly affected by the annual population frequency of androchrome females as well as by the frequencies of the two other female morphs (table 1 ; all $P<.001$; mean number of eggs $=216.9 ; \mathrm{SE}=$ 5.07).

We used these empirically estimated selection coefficients to explore the future frequencies of the morphs and the maintenance and stability of the polymorphism using our population genetic selection model (see "Methods" for details). This revealed that the current pattern and strength of frequency-dependent selection on female fecundities will maintain all three morphs indefinitely (fig. $1 A, 1 B)$. When all selection coefficients are at their mean values, the system has an internal stable equilibrium at the point $\left(P_{\mathrm{A}}=.1304, P_{\mathrm{I}}=.6759, P_{\mathrm{O}}=.1937\right)$, denoted by a black dot in figure $1 \mathrm{~A}$. A formal stability analysis revealed that at this point, the dominant eigenvalue of the Jacobian of the system (eq. [9]) is $\lambda=0.95$, which ensures stability.
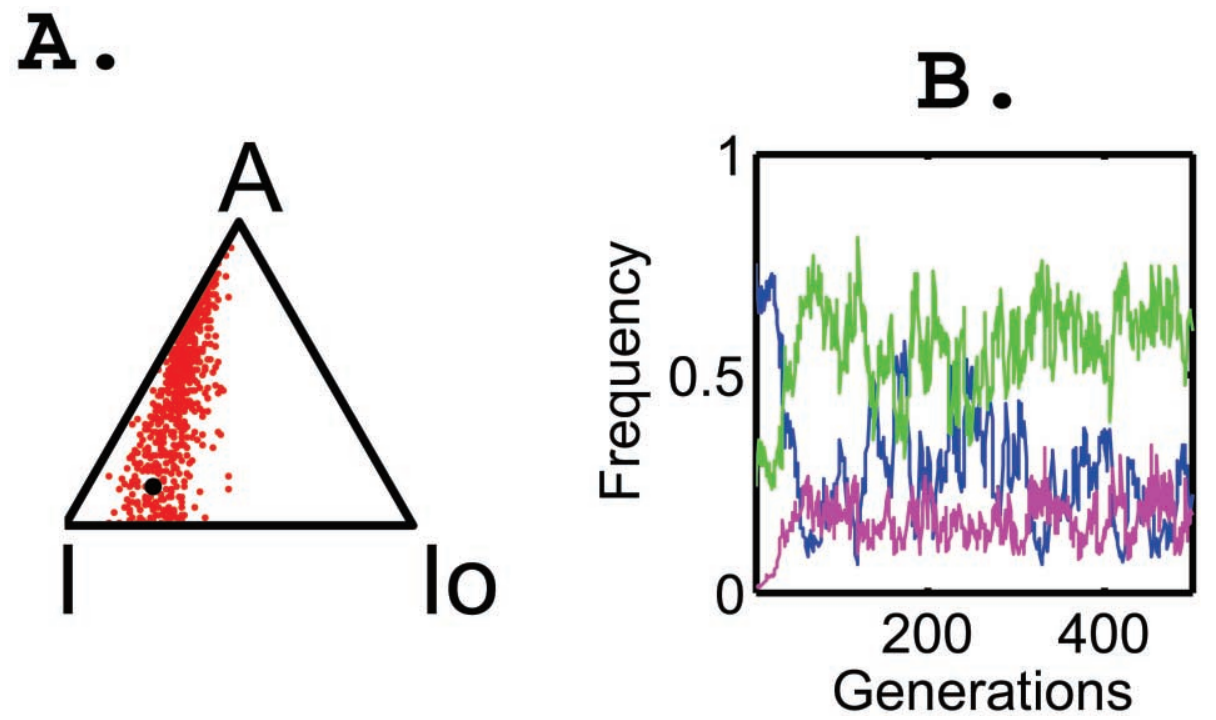

Figure 1: Results from simulations of a frequency-dependent selection model in Ischnura elegans. A, Equilibrium morph frequencies predicted from fecundity data. Any interior point in the triangle represents a frequency combination of the three color morphs. Distances from vertices are proportional to the frequency of each morph, and the top apex represents fixation of androchrome females $\left(P_{\mathrm{A}}=1\right)$. The mean multiple regression coefficients predict coexistence of all morphs with the following proportions: infuscans $=0.68$, infuscans-obsoleta $=019$, androchrome $=0.13$ (black dot). To visualize the effect of variation in the regression coefficients, the red dots depict 1,000 bootstrapped eqilibria, where we used empirically estimated variances to resample regression coefficients (see "Methods"). B, Predicted frequencies of the three morphs during 500 generations, when morph fecundities are subject to environmental noise (see "Material and Methods" for details of how environmental noise was empirically estimated). Green $=$ infuscans, blue $=$ androchrome, and pink $=$ infuscans-obsoleta . 
A

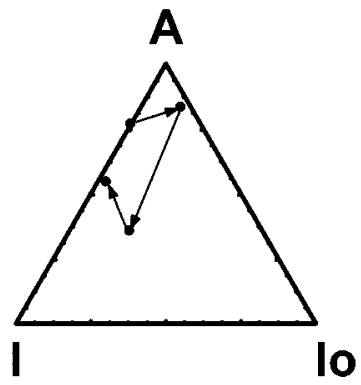

B

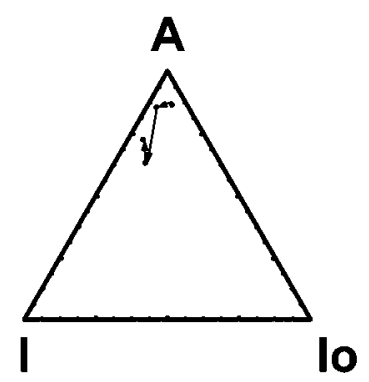

Figure 2: Ternary plots demonstrating observed morph frequency changes in two Ischnura populations in southern Sweden during 20002003 (four generations). In both populations, there were significant changes in the morph frequencies between the years. A, Hofterupssjön: $\chi^{2}=26.172, \mathrm{df}=6, P<.001 . B$, Flyinge 30 A3: $\chi^{2}=14.439$, df $=6$, $P=.025$.

We also found three stable boundary equilibria, but because of space limitations, we do not present them here because they do not describe coexistence of all morphs. To investigate how the variance of the regression coefficients influenced the result, we derived 10,000 equilibria by bootstrapping, where each time we resampled the coefficients from normal distributions with means and variances as in table 1 . One thousand of these are shown in red in figure $1 A$. In $30 \%$ of the cases, the androchrome morph was lost, but $70 \%$ of the bootstrapped equilibria comprised a combination of all three morphs at frequencies larger than the arbitrary cutoff point of 0.000001 . The mean frequencies among these $70 \%$ were $P_{\mathrm{A}}=.3832$, $P_{\mathrm{I}}=.5562, P_{\mathrm{O}}=.0606$.

Incorporating empirically estimated morph-specific fecundity variance ("environmental noise"; see "Material and Methods") results in rapid morph frequency changes between years (fig. $1 B$ ) in a frequency region in which infuscans is the most common morph, followed by androchromes and infuscans-obsoleta (fig. 1A).

Observed morph frequencies in nature differed significantly between populations $\left(\chi^{2}=66.955, \mathrm{df}=18, P<\right.$ $.001)$ and years $\left(\chi^{2}=33.066, \mathrm{df}=6, P<.001\right)$, and there was also significant heterogeneity between populations in the year-to-year changes in morph frequencies (year $x$ population: $\chi^{2}=116.046$, df $=54, P<.001$ ). Observed morph frequency changes for two populations across four years (generations) are shown in figure 2. Observed androchrome frequencies across all years and populations (mean $=0.74, \quad \mathrm{SE}=0.017, \quad$ range $=0.36-1.00)$ were higher than predicted in our model, which may be because our parameter estimates were obtained from data on one fitness component (fecundity), not total fitness. We note, however, that the predicted morph frequencies from our model fit well into the observed morph frequencies among populations of the closely related species Ischnura graellsii (Cordero 1990). Data on developmental rates indicate that there are also differences between morphs during the larval part of the life cycle, which may partly explain the quantitative discrepancy between predicted and observed morph frequencies at equilibrium (J. Abbott and E. I. Svensson, unpublished data).

However, even if fecundity is only one component of fitness, it is obviously sufficient to explain the maintenance of this polymorphism by frequency-dependent selection, and additional viability differences between morphs are unlikely to change our major conclusions. Furthermore, the polymorphism is maintained even when incorporating the effects of empirically estimated, morph-specific environmental noise (e.g., variable weather conditions; fig. 1B). This indicates the polymorphism is maintained under a broad range of biotic and abiotic environmental conditions.

\section{Discussion}

The results in this study indicate that sexual conflict over mating may be a frequency-dependent process in which the common female phenotypes face fitness disadvantages. These fitness disadvantages of common morphs arise because males form a search image directed toward the common morph (Van Gossum et al. 1999), which should lead to increased male mating harassment of the common morphs (Fincke 2004). This in turn should translate into a higher frequency of copulating females of the common morph in the field, a prediction that was confirmed in this study (table 2). A higher mating rate and higher degree of male mating harassment of the common morphs should then result in lowered fecundity of the common morphs, leading to frequency-dependent female fecundities (table 1).

These findings, although consistent with theoretical expectations, are not the only possible outcome of a sexual conflict over mating rate. Male mating harassment could have favored the evolution female counteradaptations (Arnqvist and Rowe 2002a), for instance, morph-specific rejection behaviors (Fincke 1997). If such morph-specific female counteradaptations had evolved in Ischnura elegans, we would not necessarily expect to find any differences between morphs in their field copulation rates. However, such mating-avoidance mechanisms are likely to be costly, which should constrain their evolution and lead to a situation where females accept costly copulations and actual (realized) mating rates in nature are above the female fecundity or survival optimum (Arnqvist and Nilsson 2000). 
The data in this study support such a scenario and suggest that male mating harassment of female morphs has apparently not been counteracted by the evolution of sufficiently strong mating-avoidance mechanisms in females because both morph-specific copulation probabilities and female fecundities are frequency dependent (tables 1, 2). The frequency-dependent fitness effects in the female morphs could potentially have arisen through other means than male mating harassment of common morphs, such as through female-female interactions. However, in the absence of any direct evidence for such intrasexual interactions, we suggest that the effects are indeed caused by intersexual mating interactions.

Some previous theoretical models that have incorporated frequency dependence suggest that it could affect the dynamics of sexual conflict and speciation (Gavrilets 2000). The Ischnura polymorphism is dynamic because the relative fecundity of the different color morphs changes between years, and as a consequence, the morph frequencies rapidly fluctuate between generations. Sexual conflict in this system thus plays a conservative role in maintaining a female polymorphism in most populations (figs. 1, 2). This runs counter to some recent theoretical models (Gavrilets 2000) and empirical studies (Rice 1996; Arnqvist et al. 2000; Martin and Hosken 2003) that suggest that sexual conflict may play a more creative role, enhancing the rate of population divergence through coevolutionary arms races and sexual runaway processes (Rice and Holland 1997; Gavrilets 2000). Our data are instead more consistent with other models (Parker and Partridge 1998; Gavrilets and Waxman 2002), empirical data from guppies (Magurran 1998), and recent comparative work (Gage et al. 2002), which all suggest that sexual conflict could instead constrain population divergence, for example, by maintaining a polymorphism and thereby preventing fixation and monomorphism.

Frequency-dependent male mating harassment of females may actually be a general ecological mechanism through which sexual conflict constrains rather than enhances population divergence. It is also possible that different genetic architectures underlying traits under sexual conflict could partly explain the discrepancy between our findings and the previous theoretical models because these assumed many loci of small effect rather than a majoreffect locus, as in the case of the color polymorphism in Ischnura.

A recent theoretical model (Gavrilets and Waxman 2002) suggests that sexual conflict can promote the splitting of females into genetically distinct groups (i.e., promote and maintain a polymorphism) as an alternative to perpetual sexual coevolutionary arms races and subsequent speciation (Gavrilets 2000). Female diversification brings the coevolutionary arms race between males and females to an end (Gavrilets and Waxman 2002), and this model may be applicable to the female color polymorphism in Ischnura. Our data indicate that sexual conflict maintains all three morphs in all populations and prevents any of them from going to fixation by genetic drift or local selection, thereby constraining population divergence in morph frequencies (figs. 1, 2). In contrast, if frequency selection was not operating or was only a weak force, one would expect the different populations to become fixed (monomorphic) for different morphs, either by genetic drift or through local frequency-independent selection generated by population differences in ecology. In general, if sexual conflict through male mating harassment leads to a rare-morph advantage, a more likely outcome is stabilization of morph frequencies or genetic evolutionary cycles (Sinervo and Lively 1996; Sinervo et al. 2000) rather than ever-escalating arms races and speciation (Rice and Holland 1997).

Recent empirical work on several different taxa (Bergsten et al. 2001; Arnqvist and Rowe 2002a, 2002b; Rowe and Arnqvist 2002) suggests that sexual conflict may have important evolutionary consequences in natural populations. The results in this study add further evidence to this but do also suggest that the evolutionary dynamics of sexual conflict may include a strong frequency-dependent component. We encourage other workers in this area to further explore the generality of these findings, with the aim to understand whether, when, and how often frequency-dependent selection plays a major role in the evolution of male-female mating interactions.

\section{Acknowledgments}

We are grateful to A. Forsman, A. Hargeby, D. Hasselqvist, J. Johansson, L. B. Pettersson, J. Ripa, L. Rowe, M. Stjernman, and several anonymous reviewers for constructive criticisms and comments on the first drafts of this manuscript. This study was financially supported by grants from the Swedish Research Council, Vetenskapsrådet (to E.I.S. and R.H.), and from the Royal Swedish Academy of Sciences and Oscar and Lilli Lamms Stiftelse (to E.I.S.).

\section{Literature Cited}

Ågren, J., and L. Ericson. 1996. Population structure and morphspecific fitness differences in tristylous Lythrum salicaria. Evolution 50:126-139.

Andrés, J. A., R. A. Sánchez-Guillen, and A. Cordero Rivera. 2000. Molecular evidence for selection on female color polymorphism in the damselfly Ischnura graellsii. Evolution 54:2156-2161.

- 2002. Evolution of female colour polymorphism in damselflies: testing the hypotheses. Animal Behaviour 63:677-685.

Arnqvist, G. 1998. Comparative evidence for the evolution of genitalia by sexual selection. Nature 393:784-786.

Arnqvist, G., and T. Nilsson. 2000. The evolution of polyandry: mul- 
tiple mating and female fitness in insects. Animal Behaviour 60: $145-164$.

Arnqvist, G., and L. Rowe. 2002a. Antagonistic coevolution between the sexes in a group of insects. Nature 415:787-789.

2002b. Correlated evolution of male and female morphologies in water striders. Evolution 56:936-947.

Arnqvist, G., M. Edwardsson, U. Friberg, and T. Nilsson. 2000. Sexual conflict promotes speciation in insects. Proceedings of the National Academy of Sciences of the USA 97:10460-10464.

Askew, R. R. 1988. The dragonflies of Europe. Harley, Colchester.

Bergsten, J., A. Toyra, and A. N. Nilsson. 2001. Intraspecific variation and intersexual correlation in secondary sexual characters of three diving beetles (Coleoptera: Dytiscidae). Biological Journal of the Linnean Society 73:221-232.

Cooper, G., P. L. Miller, and P. W. H. Holland. 1996. Molecular genetic analysis of sperm competition in the damselfly Ischnura elegans (Vander Linden). Proceedings of the Royal Society of London B 263:1343-1349.

Corbet, P. S. 1999. Dragonflies: behaviour and ecology of Odonata. Harley, Colchester.

Cordero, A. 1990. The inheritance of female polymorphism in the damselfly Ischnura graellsii (Rambur) (Odonata: Coenagrionidae). Heredity 64:341-346.

—. 1992. Density-dependent mating success and colour polymorphism in females of the damselfly Ischnura graellsii (Odonata: Coenagrionidae). Journal of Animal Ecology 61:769-780.

Cordero, A., S. S. Carbone, and C. Utzeri. 1998. Mating opportunities and mating costs are reduced in androchrome female damselflies, Ischnura elegans. Animal Behaviour 55:185-187.

Draper, N. R., and H. Smith. 1998. Applied regression analysis. Wiley, New York.

Eckert, C. G., and C. H. Barrett. 1995. Style morph ratios in tristylous Decodon verticillatus (Lythraceae): selection vs. historical contingency. Ecology 76:1051-1066.

Fincke, O. M. 1986. Lifetime reproductive success and the opportunity for selection in a nonterritorial damselfly (Odonata, Coenagrionidae). Evolution 40:791-803.

- 1994. Female color polymorphism in damselflies: failure to reject the null hypothesis. Animal Behaviour 47:1249-1266.

- 1997. Conflict resolution in the Odonata: implications for understanding female mating patterns and female choice. Biological Journal of the Linnean Society 60:201-220.

- 2004. Polymorphic signals of harassed female odonates and the males that learn them support a novel frequency-dependent model. Animal Behaviour 67:833-845.

Gage, M. J. G., G. A. Parker, S. Nylin, and C. Wiklund. 2002. Sexual selection and speciation in mammals, butterflies and spiders. Proceedings of the Royal Society of London B 269:2309-2316.

Gavrilets, S. 2000. Rapid evolution of reproductive barriers by sexual conflict. Nature 403:886-889.

Gavrilets, S., and D. Waxman. 2002. Sympatric speciation by sexual conflict. Proceedings of the National Academy of Sciences of the USA 99:10533-10538.

Gendron, R. P. 1987. Models and mechanisms of frequency-dependent predation. American Naturalist 130:603-623.

Hartl, D. L., and A. G. Clark. 1997. Principles of population genetics. Sinauer, Sunderland, MA.

Holland, B., and W. R. Rice. 1999. Experimental removal of sexual selection reverses intersexual coevolution and removes a repro- ductive load. Proceedings of the National Academy of Sciences of the USA 96:5083-5088.

Huey, R. B., G. W. Gilchrist, M. L. Carlson, D. Berrigan, and L. Serra. 2000. Rapid evolution of a geographic cline in size in an introduced fly. Science 287:308-309.

Kingsolver, J. G., H. E. Hoekstra, J. M. Hoekstra, D. Berrigan, S. N. Vignieri, C. E. Hill, A. Hoang, P. Gibert, and P. Beerli. 2001. The strength of phenotypic selection in natural populations. American Naturalist 157:245-261.

Lande, R., and S. J. Arnold. 1983. The measurement of selection on correlated characters. Evolution 37:1210-1226.

Magurran, A. E. 1998. Population differentiation without speciation. Philosophical Transactions of the Royal Society of London B 353: 275-286.

Martin, O. Y., and D. J. Hosken. 2003. The evolution of reproductive barriers through sexual conflict. Nature 423:979-981.

Parker, G. A., and L. Partridge. 1998. Sexual conflict and speciation. Philosophical Transactions of the Royal Society of London B 353: 261-274.

Reznick, D., F. H. Shaw, F. H. Rodd, and R. G. Shaw. 1997. Evaluation of the rate of evolution in natural populations of guppies (Poecilia reticulata). Science 275:1934-1937.

Rice, W. R. 1996. Sexually antagonistic male adaptation triggered by experimental arrest of female evolution. Nature 381:232-234.

Rice, W. R., and B. Holland. 1997. The enemies within: intergenomic conflict, interlocus contest evolution (ICE), and the intraspecific Red Queen. Behavioral Ecology and Sociobiology 41:1-10.

Robertson, H. M. 1985. Female dimorphism and mating behaviour in a damselfly, Ischnura ramburi: females mimicking males. Animal Behaviour 33:805-809.

Robinson, J. V., and R. Allgeyer. 1996. Covariation in life-history traits, demographics and behaviour in ischnuran damselflies: the evolution of monandry. Biological Journal of the Linnean Society 58:85-98.

Rowe, L. 1992. Convenience polyandry in a water strider: foraging conflicts and female control of copulation frequency and guarding duration. Animal Behaviour 44:189-202.

Rowe, L., and G. Arnqvist. 2002. Sexually antagonistic coevolution in a mating system: combining experimental and comparative approaches to address evolutionary processes. Evolution 56:754-767.

Sinervo, B., and C. M. Lively. 1996. The rock-paper-scissors game and the evolution of alternative male strategies. Nature 380:240243.

Sinervo, B., and E. Svensson. 2002. Correlational selection and the evolution of genomic architecture. Heredity 16:948-955.

Sinervo, B., E. Svensson, and T. Comendant. 2000. Density cycles and an offspring quantity and quality game driven by natural selection. Nature 406:985-988.

Sirot, L. K., and H. J. Brockmann. 2001. Costs of sexual interaction to females in Rambur's forktail damselfly, Ischnura ramburi (Zygoptera: Odonata). Animal Behaviour 61:415-424.

StatSoft. 2003. STATISTICA (data analysis software system). Version 6. Tulsa, OK. http://www.statsoft.com.

Svensson, E. I., and B. Sinervo. 2004. Spatial scale and temporal component of selection in side-blotched lizards. American Naturalist 163:726-734.

Svensson, E., B. Sinervo, and T. Comendant. 2001a. Condition, genotype-by-environment interaction and correlational selection in lizard life-history morphs. Evolution 55:2053-2069.

2001b. Density-dependent competition and selection on im- 
mune function in genetic lizard morphs. Proceedings of the National Academy of Sciences of the USA 98:12561-12565.

Svensson, E. I., L. Kristoffersen, K. Oskarsson, and S. Bensch. 2004. Molecular population divergence and sexual selection on morphology in the banded demoiselle (Calopteryx splendens). Heredity 93:423-433.

Van Gossum, H., R. Stoks, E. Matthysen, F. Valck, and L. De Bruyn. 1999. Male choice for female colour morphs in Ischnura elegans (Odonata, Coenagrionidae): testing the hypotheses. Animal Behaviour 57:1229-1232.

Van Gossum, H., R. Stoks, and L. De Bruyn. 2001. Frequencydependent male mate harassment and intra-specific variation in its avoidance by females of the damselfly Ischnura elegans. Behavioral Ecology and Sociobiology 51:69-75.

Wong, A., M. L. Smith, and M. R. Forbes. 2003. Differentiation between subpopulations of a polychromatic damselfly with respect to morph frequencies, but not neutral genetic markers. Molecular Ecology 12:3505-3513.

Yoshida, T., L. E. Jones, S. P. Ellner, G. F. Fussman, and N. G. Hairston Jr. 2003. Rapid evolution drives ecological dynamics in a predatorprey system. Nature 424:303-306.

Editor: Jonathan B. Losos Associate Editor: Allen J. Moore 